\title{
UMA BREVE INTRODUÇÃO \\ À FILOSOFIA DA CIÊNCIA EM PRÁTICA
}

\section{Luana Poliseli ${ }^{1}$}

\begin{abstract}
RESUMO
A filosofia da ciência estuda a ciência, o modus operandi da ciência e o conhecimento científico. Convencionalmente, as investigações filosóficas desse campo se debruçam principalmente sobre aspectos metafísicos, epistemológicos e metodológicos da produção de conhecimento nas ciências. Muito embora seja tradicionalmente subdividida em filosofia geral das ciências e filosofia das ciências especiais, a filosofia da ciência de uma forma geral ainda se mantém distante das práticas científicas propriamente ditas. Para suprir tal demanda surge uma terceira subdivisão, ainda embrionária, a filosofia da ciência em prática. Este artigo apresenta uma breve introdução à filosofia da ciência em prática e à Sociedade de Filosofia da Ciência em Prática, discute seus objetivos e estratégias metodológicas, bem como seu engajamento social e empírico. Espero que, com esse artigo, seja possível trazer uma breve noção sobre a diversidade de investigações e as possibilidades de uma filosofia da ciência que pode ir para além do escopo meta-analítico, que pode ser empiricamente engajada e socialmente informada.
\end{abstract}

Palavras-chave: Prática Científica. Virada Empírica. Pragmatismo. Naturalismo. Sociedade de Filosofia da Ciência em Prática. Filosofia-da-Ciência em Prática.

\begin{abstract}
Philosophy of science studies science and the production of scientific knowledge. Usually, philosophical investigations of this field focus mainly on metaphysical, epistemological and methodological aspects of science. Despite being divided into general philosophy of science and philosophy of special sciences, philosophy of science, in a general way, is still distant from scientific practice per se. In order to fill this gap, a third subfield has emerged, philosophy of science in practice. This article provides a brief introduction to the philosophy of science in practice and to the Society for Philosophy of Science in Practice. It discusses its goals, methods, its social and empirical engagement. It is expected that this article will shed light on the diversity and possibility of investigations for a philosophy of science

\footnotetext{
${ }^{1}$ Instituto Nacional de Ciência e Tecnologia em estudos Inter e Transdisciplinares em Ecologia e Evolução. Laboratório de Ensino, Filosofia e História da Biologia, UFBA.

E-mail: luapoliseliramos@gmail.com.
} 
beyond meta-analysis, in other words, that are empirically engaged and socially informed.

Keywords: Scientific Practice. Empirical Turn. Pragmatism. Naturalism. Society for Philosophy of Science in Practice. Philosophy-of-Science in Practice.

To see a World in a Grain of Sand

And a Heaven in a Wild Flower Hold Infinity in the palm of your hand And Eternity in an hour Auguries of Innocence, William Blake Pickering Manuscript, 1863

\section{Introdução}

A filosofia da ciência é um ramo da filosofia que se ocupa em estudar a ciência. Frequentemente, é subdividida em filosofia geral da ciência e filosofia das ciências especiais. Ambos os campos se dedicam a investigar a natureza e a produção do conhecimento científico através de investigações ontológicas, epistemológicas, metodológicas e éticas da ciência. Tradicionalmente, a filosofia da ciência tem se debruçado em uma descrição do conhecimento científico de acordo com uma relação dual entre mundo e conhecimento (BOON, 2017). Tais enfoques podem ser realizados tanto de uma perspectiva geral quanto contextualizadas em disciplinas específicas, como a física, biologia, química, economia, medicina, entre outras. Muito embora esta longa tradição da literatura filosófica sobre a natureza das explicações científicas ajude a revelar características cruciais sobre as explicações entre as ciências (CRAVER, 2007), a maioria das investigações em filosofia da ciência continua quase isolada da prática científica per se (ANKENY et al., 2011), ou seja, os estudos de caso são majoritariamente teóricos ou reconstruções históricas.

Com o intuito de fornecer um olhar mais atento ao lado empírico da empreitada científica, surge a filosofia da ciência em prática. Esta área, ainda embrionária, reformula, em termos de atividades, as questões abstratas ou puramente teóricas sobre a prática científica advindas dos tradicionais debates filosóficos (e.g. verdade, justificação, evidência, observação, dados, experimentação, etc.) e examina os objetivos subjacentes das atividades 
associadas à ciência focando não somente em considerações epistemológicas mas também nos valores, normas e ideais inerentes à busca do conhecimento científico (ANKENY et al., 2011). Sendo assim, são dois os objetivos deste artigo. Primeiro, o de trazer uma breve introdução à filosofia da ciência em prática, seus objetivos e procedimentos analíticos. E segundo, incitar futuros debates e instigar novas estratégias de ação para uma filosofia da ciência que vá, também, para além da esfera meta-analítica.

Este artigo estrutura-se da seguinte forma: a seção 1 introduz brevemente as tradicionais subáreas filosofia geral da ciência e filosofia das ciências especiais (individuais ou particulares) com um leve enfoque na chamada 'virada prática'. A seção 2 apresenta a filosofia da ciência em prática, manifesta as missões da Sociedade de Filosofia da Ciência em Prática e contextualiza alguns temas de pesquisas predominantes que estão relacionados à dualidade de abordagens apresentadas nesse campo. Por último, é realizada uma breve consideração, que se limita ao estímulo e prospectos investigativos desta área.

\section{A filosofia geral da ciência, a filosofia das ciências especiais e a virada prática}

Atualmente, percebemos um rápido crescimento na filosofia da ciência refletida no aumento da quantidade de pesquisadores, associações e institutos dedicados à temática, que pode ser associado ao próprio crescimento da ciência e à consolidação do paradigma científico no final do século XX e início do século $\mathrm{XXI}^{2}$ (ANKENY et al., 2011).

Até meados de 1970, a filosofia da ciência consistia em um campo pequeno com poucas revistas, edições especiais e trabalhos monográficos (RADDER, 2012). A filosofia da ciência era usualmente concebida como filosofia geral da ciência. E a física era assumida como sendo a ciência modelo - com subjacentes ideais de unificação/redução. Na década de 1970, o

\footnotetext{
${ }^{2}$ Vale salientar que este artigo não pretende discorrer sobre os fatores sociais e históricos associados ao desenvolvimento da ciência e filosofia da ciência e, especialmente, não reflete uma ordem cronológica restrita. Reconheço que essa tentativa de sumarização é, em certa medida ingênua, e só arranha a superfície deste vasto oceano de discussões que permeiam o desenvolvimento dessas áreas do saber. Para informações sobre história da ciência e historiografia da filosofia da ciência ver FRANK (1957), FODOR (1974), LADYMAN (2001, 2019), BRAGA et al., (2003, 2004, 2005, 2007), PSILLOS (2012) entre outros.
} 
proeminente foco em áreas como as ciências cognitivas e temas como os limites da unificação, levaram ao desenvolvimento de uma filosofia das ciências especiais (FODOR 1974). Assim, houve uma fragmentação e subsequente especialização: jornais e revistas se multiplicaram focando em disciplinas, temas e metodologias cada vez mais específicas (RADDER, 2012). A tradicional subdivisão em filosofia geral da ciência e filosofia das ciências especiais (particulares ou individuais) (BARBEROUSSE et al., 2011), pode ser interpretada como um reflexo desse processo.

A filosofia geral das ciências lida, de uma forma geral, com problemas metafísicos e epistemológicos que perpassam todas as ciências e.g. o que é uma explicação e compreensão científica (HEMPEL \& OPPENHEIM, 1948; KITCHER, 1981; SALMON, 1984; DE REGT, 2017; KHALIFA, 2017, etc.); quais são as leis da natureza (ver CID, 2019), quais são os tipos naturais, se devemos adotar realismo científico ou não (HACKING, [1983]2012; DUPRÉ, 1993; PSILLOS, 1999, etc., ver também OLIVEIRA, 2019); o que é objetividade (DASTON \& GALISON, 2010); e discussões metodológicas, de demarcação e progresso (POPPER, [1959]1993; KUHN, [1962]1997; SHAPERE, 1984; LAKATOS, 1987; FEYERABEND, [1987]2010, entre outros). Já a filosofia das ciências especiais examinam esses problemas filosóficos em relação às ciências individuais, bem como investigam os problemas inerentes de cada ciência específica, como a biologia, geologia, psicologia, economia, etc; e.g. o que é uma explicação evolutiva (MAYR, [1982]1998); se existem leis em biologia como existem na física (CAPONI, 2014); se quarks, átomos, indivíduos, espécies, organismos existem na natureza ou se são modelos teóricos (para modelos ver MORGAN \& MORRISON, 1999; KNUUTTILA, 2011); como a redução ocorre na neurociência (CRAVER, 2007), entre outros. Os debates que abarcam ambas as esferas podem ser debatidos através da relação entre ciência (subsequentes disciplinas e produtos) e valores (ver LACEY, 1998, 2008, 2010; LONGINO, 1990; para entrevista com Alison Wylie e Helen Longino ver KOIDE et al. 2014 e AYMORÉ et al. 2017, respectivamente), e a comodificação da ciência (RADDER, 2010; DE OLIVEIRA, 2013). Tais investigações permitem aproximar as discussões filosóficas da ciência com a realidade e práticas sociais. 
Radder (2012) aponta que o aumento na especialização e fragmentação da filosofia da ciência reduziu seu impacto para fora da esfera direta de seus praticantes, e chama a atenção de que, inclusive, fora designada por alguns como a "Sibéria da filosofia". Apesar dos inúmeros avanços teóricos nas investigações sobre as práticas da ciência refletida por essas subáreas, a filosofia da ciência se manteve afastada do contexto da prática científica; por exemplo, grande parte das análises filosóficas eram baseadas em interpretações dos resultados já publicados e teorias científicas aceitas, ao invés de ser engajada com cientistas in vivo e analisar o real processo de experimentação, coleta de dados, etc. Diversos foram os esforços em defesa dessa aproximação, a tão chamada "virada prática" nas décadas de 1980 advogou que as investigações filosóficas, históricas e sociais da ciência deveriam considerar, nas práticas científicas, detalhes meticulosos que reconhecessem suas dimensões sociais, materiais e psicológicas (SOLER et al., 2014; PICKERING, 1992).

Outros argumentos filosóficos sistemáticos a favor de um enfoque forte nas distintas práticas da ciência também existiram com os pós-Kuhnianos, os naturalistas e os pós-modernistas (RADDER, 2012). Os pós-Kuhnianos defendiam que a ciência não poderia ser compreendida somente a partir de uma perspectiva de poltrona (para armchair philosophy ver BRYSON, 2009) posto que a filosofia da ciência precisava se atentar aos detalhes das práticas históricas dos cientistas. Já os naturalistas questionaram a distinção entre filosofia e ciência argumentando que, a fim de compreender as atividades científicas, tais como observação ou raciocínio teórico, seria necessária uma abordagem à luz das ciências cognitivas. E por fim, os pós-modernistas (ver SHINN \& RAGOUET, 2008) avançaram com críticas fundamentais sobre uma epistemologia geral da ciência, de teorias universais da racionalidade, e de metodologias científicas compreensíveis (RADDER, 2012).

Fora da filosofia da ciência também foi possível ver certos estímulos nessa mesma direção, por exemplo, filósofos declaradamente pragmatistas (como Charles S. Peirce e John Dewey), da linguagem comum e seguidores do Wittgenstein tardio se esforçaram em buscar verdade e significado através das práticas e instrumentos (ver STERN, 2003). Já aqueles nas tradições de filosofia continental também enfatizaram a necessidade de se considerar 
a experiência e a prática, bem como rejeitaram algumas tradições positivistas as quais viam a ciência como excessivamente privilegiada, tendo o progresso científico como certo (ANKENY et al., 2011; para significados políticos das tradições positivistas ver OLIVEIRA, 2002). Assim, apesar dos esforços da filosofia naturalista da ciência em defender enfaticamente a necessidade de estudar as práticas científicas em abordagens integrativas de história, filosofia e sociologia da ciência, iniciativas externas à filosofia da ciência também existiram.

Com esta virada, o interesse nas práticas científicas continuou aumentando. No entanto, de uma forma geral, a filosofia da ciência permaneceu focada na relação das teorias científicas com o mundo, enquanto os estudos históricos e sociais da ciência e tecnologia encararam as práticas científicas como produção humana (ANKENY et al., 2011). Ambas áreas produziram trabalhos indubitavelmente marcantes, como Galison (1987), Pickering (1992), Galison \& Stump (1996), Bourdieu ([1993]2004), entre outros. Mas se o objetivo é entender e explorar as premissas e métodos subjacentes da ciência, ambos os escopos estavam limitados não só por negligenciarem as perspectivas e abordagens relevantes para o desenvolvimento de um quadro mais completo da ciência, como também por negligenciarem os processos pelos quais levaram às conclusões e aos produtos da própria ciência (ANKENY et al., 2011). Ecoando essa preocupação, surge uma terceira subárea, a filosofia da ciência em prática, e será elaborada a seguir.

\section{A Filosofia da Ciência em Prática}

Com algumas poucas exceções, grande parte das investigações filosóficas são quase exclusivamente inferências abstratas (MURCHO, 2006; QUINHONE \& COSTA, 2010). As análises sobre a ciência realizadas pela tradicional filosofia anglófona têm sido excessivamente limitadas pelo hábito de ver a ciência enquanto um corpo de proposições. Como consequência, tais investigações focam no valor real dessas proposições e na relação lógica entre elas. Devido ao objeto de discussão nessa filosofia da ciência ter sido teorias enquanto estruturas proposicionais organizadas, a experimentação e 
outras dimensões das ciências que são não-verbais e não-proposicionais foram negligenciadas dessas investigações filosóficas (CHANG, 2014).

No contrafluxo, em 2005 na conferência Philosophical Perspectives on Scientific Understanding em Amsterdam, surge a ideia para a criação da Sociedade de Filosofia da Ciência em Prática (SPSP) (com posterior introdução e convite à comunidade em 2006 na PSA - Philosophy of Science Association). A SPSP chama a atenção da filosofia para as práticas científicas e se dedica a incentivar uma filosofia da ciência baseada em um arcabouço analítico que considere a teoria, a prática e o mundo simultaneamente, e nunca um isolado do outro (ANKENY et al., 2011). Para entender as implicações dessa área é necessário primeiro compreender como a SPSP define "prática":

Prática consiste em atividades organizadas ou reguladas de forma a atingir certos objetivos. Desta forma, a epistemologia da prática deve elucidar quais tipos de atividades são necessárias para a geração de conhecimento. Os debates tradicionais em epistemologia (relacionados à verdade, fato, crenças, observação, explicação, justificação, evidência, etc.) podem ser redefinidos em termos de atividades. De uma forma similar, tratamentos baseados em prática irão esclarecer questões sobre modelos, medidas, experimentos, etc., que têm surgido com proeminência em anos recentes e que consideram o trabalho científico real (Sociedade de Filosofia da Ciência em Prática - SPSP) ${ }^{3}$.

A partir dessa definição, focar na prática permitiria a filosofia da ciência voltar a questões fundamentais que ficaram gradativamente negligenciadas de um olhar empírico. Ademais, examinar os objetivos subjacentes das atividades associadas à ciência também permite debater valores, normas, e ideais inerentes à busca de conhecimento científico (ANKENY et al., 2011), e facilitam, assim, o diálogo para com outras investigações sobre ciência, tecnologia e sociedade.

\footnotetext{
3 "Practice consists of organized or regulated activities aimed at the achievement of certain goals. Therefore, the epistemology of practice must elucidate what kinds of activities are required in generating knowledge. Traditional debates in epistemology (concerning truth, fact, belief, certainty, observation, explanation, justification, evidence, etc.) may be re-framed with benefit in terms of activities. In a similar vein, practice-based treatments will also shed further light on questions about models, measurement, experimentation, etc., which have arisen with prominence in recent years decades from considerations of actual scientific work (Society for Philosophy of Science in Practice - SPSP)", trecho retirado da plataforma: https://www.philosophy-science-practice.org.
} 
Alguns aspectos da filosofia da ciência em prática são destacados no trabalho de Ankeny et al., (2011), mas podem ser encontrados em mais detalhes na plataforma da Sociedade de Filosofia da Ciência em Prática (SPSP) ${ }^{4}$, e seguem.

Primeiro, a preocupação não é somente a aquisição e validação do conhecimento, mas também o seu uso, ou seja, não é somente sobre como o conhecimento preexistente é aplicado para fins práticos, mas também sobre como o conhecimento propriamente dito é moldado pelos seus usos intencionais.

Segundo, enfatiza-se como os artefatos humanos, tais como modelos conceituais e instrumentos laboratoriais, fazem a mediação entre teorias e mundo. Além do mais, busca-se elucidar o papel desses artefatos na formação da prática científica.

Terceiro, a noção de prática científica não deve ser distorcida por visões assimétricas de determinadas áreas da ciência que são predominantes. É possível integrar tais áreas levando a atenção a outros campos como engenharia, economia, ciências humanas e sociais, ciências médicas, bem como outras áreas relativamente negligenciadas nas ciências naturais.

Quarto, o objetivo em si é que a filosofia da ciência seja gradativamente vista como relevante e útil para praticantes científicos. Sendo assim, é crucial refletir uma disciplina integrada onde a participação de cientistas praticantes, engenheiros e tomadores de decisões são estimuladas, não somente para aprender sobre as suposições subjacentes e as implicações dessas práticas, mas também para aprender com as próprias práticas e os praticantes.

\subsection{Dualidade de abordagens e aplicações}

A Sociedade de Filosofia da Ciência em Prática está interessada numa filosofia da ciência a partir de uma perspectiva prática (KOSOLOSKY, 2012). Dispõe, assim, da prática científica como seu objeto de estudo. No entanto, não possui nenhuma metodologia específica ou protocolo geral a seguir para atingir seu objetivo de estudar a ciência. Os instrumentos

\footnotetext{
${ }^{4}$ As quatro missões da Sociedade foram traduzidas e retirada da plataforma: https://
} www.philosophy-science-practice.org. 
utilizados para tanto advêm de outras áreas como história, sociologia, psicologia mais especificamente da história da ciência, ciência-tecnologia-e-sociedade, sociologia da ciência entre outros (BOON, 2017). A falta de uma metodologia geral ou de uma abordagem específica não faz a filosofia da ciência em prática menos válida, ao contrário, os instrumentos supracitados em sua natureza interdisciplinar constituem uma caixa de ferramentas para se atingir o objetivo de compreender como a ciência é feita. Ao invés de se apegar a um único protocolo, a filosofia da ciência em prática possui a vantagem de utilizar um set, ou uma família de abordagens, oriundos de diversos campos. O desafio, no entanto, é mapear como esses processos metodológicos podem acontecer nesta janela de oportunidades (POLISELI, 2018).

Focando nas possíveis abordagens da filosofia da ciência em prática, John Dupré na terceira conferência bienal da Sociedade de Filosofia da Ciência em Prática, em 2011 na Universidade de Exeter, trouxe uma instigante discussão sobre como estudar a prática científica. Segundo Dupré, existem duas formas possíveis, a filosofia-da-ciência em prática e a filosofia da ciência-em-prática (BOUMANS \& LEONELLI, 2013).

A filosofia-da-ciência em prática é a filosofia diretamente engajada com a pesquisa científica através da interação com cientistas sobre problemas filosóficos (e.g. estrutura lógica, implicações de resultados inesperados ou indesejados) e/ou a colaboração em questões conjuntas (e.g. o status da matemática na biologia, a confiabilidade de simulações, ou a clareza conceitual nas análises de dados). Este tipo de abordagem não requer que os filósofos se envolvam com métodos empíricos, muito embora isto possa acontecer a depender do contexto; mas a ênfase aqui é em identificar campos em comum entre a investigação filosófica e a científica (BOUMANS \& LEONELLI, 2013). Este tipo de solução de problemas é passível de ser resolvida pelos cientistas, no entanto, filósofos informados cientificamente stão inclinados a fazê-lo (KOSOLOSKY, 2012) e.g. dicotomia entre conhecimento ecológico tradicional e conhecimento ecológico acadêmico (LUDWIG \& POLISELI, 2018), debates sobre a síntese evolutiva estendida, taxonomia e/ou tipos naturais (EL-HANI \& LUDWIG, 2020), onde filósofos debatem com cientistas, mas não se envolvem em dados de coleções ci- 
entíficas. Uma filosofia da ciência engajada socialmente também se enquadra nesta abordagem uma vez que levam suas investigações para as esferas das práticas sociais e empíricas. ${ }^{5}$

Já a filosofia da ciência-em-prática é a filosofia que analisa a ciência sendo feita, i.e. o cotidiano de tudo aquilo que está associado à pesquisa científica (e.g. processo de investigação, acordos institucionais, dinâmica social entre os investigadores, etc.). Nesta abordagem, os filósofos não necessariamente colaboram com os cientistas, mas utilizam métodos empíricos derivados da história ou sociologia (e.g. etnografias, entrevistas, arquivos etc.) para obter evidências sobre o comportamento de pesquisa dos cientistas (BOUMANS \& LEONELLI, 2013). Aqui, a filosofia está engajada com as pessoas e comunidades que produzem a ciência em suas mais diversas formas, por exemplo, uma investigação que foca na natureza da experimentação explicativa e como ela se relaciona com a experimentação baseada em hipótese (KOSOLOSKY, 2012, ver entrevista com Kevin Elliot); ou então, sobre a dinâmica colaborativa de pesquisadores inseridos em investigações interdisciplinares (POLISELI \& LEITE, 2020, no prelo). Outros exemplos dessa abordagem são encontrados em estudos sobre a natureza e fluxo dos dados em data science (ver LEONELLI, 2016; LEONELLI \& TEMPINI, 2020); investigações sobre a natureza da compreensão científica (DE REGT \& DIEKS, 2005; DE REGT, 2017), entre outros.

Essas definições ajudam a compreender distintas formas de se estudar a prática científica e que não precisam, necessariamente, encerrar-se em si mesmas - ao contrário, é um ponto de partida para se discutir possibilidades metodológicas (KOSOLOSKY, 2012). A adoção de uma das abordagens em uma investigação não é, obrigatoriamente, excludente da outra. Isso permite que certa intersecção nesta dualidade seja possível. O seguinte exemplo ilustra esta dinâmica. Em um estudo de caso na ecologia, a filosofia contribui ativamente para a prática científica de construção de modelos, através da

\footnotetext{
${ }^{5}$ Algumas iniciativas interessantes podem ser indicadas. A Socially-Engaged Philosophy realizada pelo professor Martin Kusch, da Universidade de Viena. E, o consórcio SRPoiSE - Socially Relevant Philosophy of/in Science and Engineering e pode ser acessado pela plataforma http://srpoise.org/. E, no Brasil, pesquisas a serem indicadas fazem parte do Instituto Nacional de Ciência e Tecnologia em Estudos Inter- e Transdisciplinares em Ecologia e Evolução (INCT-INTREE) coordernado pelo professor Charbel El-Hani, na Universidade Federal da Bahia (site de divulgação e comunicação científica: https://darwinianas.com/).
} 
nova filosofia mecanística, para explicar um fenômeno ecológico, configurando assim filosofia-da-ciência em prática; e na sequência se afasta dessa colaboração para avaliar como o entendimento científico do cientista é atingido durante o processo de construção de modelos, configurando então filosofia da ciência-em-prática (ver POLISELI, 2018).

O que poderia ser encarado como um algoz metodológico para a filosofia da ciência em prática seria sua falta de precisão e rigor, mas isto, no entanto, pode ser contornado através de sugestões advindas da filosofia analítica, estudos em ciência, tecnologia e sociedade (CTS) e demais áreas. Segundo Murcho (2006) é ingênuo pensar que é necessário desenvolver métodos de pesquisa confiáveis para então realizar perguntas fundamentais e importantes, pois questões difíceis e puzzling conduzem o desenvolvimento de novos métodos de investigação. Uma vez que esses questionamentos cessam, também cessam esses desenvolvimentos metodológicos (MURCHO, 2006). Alguns exemplos interessantes dessa versatilidade seguem. Aufrecht (2011), analisando a distinção do "contexto de descoberta" e "contexto de justificação", examina a legitimidade das abordagens feministas para a filosofia da ciência, enquanto Soler (2011) investiga a dimensão tácita do conhecimento, argumentando que a irredutibilidade de pressuposições e habilidades corporais envolvidas nas práticas experimentais conferem certa opacidade para estas mesmas práticas (ANKENY et al., 2011). Chang (2014) utiliza os conceitos de "atividades epistêmicas" e "sistemas de práticas" como unidades de análises para construir discussões sobre ciência e desenvolve um arcabouço filosófico estruturado para investigar as práticas científicas. E Poliseli (2018) desenvolve uma caixa de ferramentas para a filosofia da ciência em prática que tem como ferramentas processos heurísticos e interdisciplinares que servem de instrumentos tanto para a filosofiada-ciência em prática como para a filosofia da ciência-em-prática.

\subsection{Rompendo barreiras disciplinares}

Atualmente, parte da ciência lida com problemas perversos (e.g. pandemias, perda de biodiversidade, mudanças climáticas, pobreza, terrorismo, etc.), para lidar com tal complexidade são requeridas cada vez mais es- 
tratégias inter- e transdisciplinares. Se o objetivo é verificar a extensão e as formas pelas quais todas as áreas da ciência são moldadas pelos usos do conhecimento gerado através da pesquisa científica, tais práticas científicas não mais se encaixam no modelo kuhniano de ciência normal disciplinar (ver ANDERSEN, 2013). É crucial então romper barreiras tradicionais entre ciências "pura", "aplicadas", "naturais", "sociais" e entre "ciência" e "tecnologia" para encorajar investigações sobre o uso desse conhecimento para fins práticos, tanto no mundo material como social (ANKENY et al., 2011).

Tendo em mente que essas demarcações são um tanto quanto fluidas, é possível perceber, então, que a filosofia da ciência em prática pode trabalhar na intersecção de distintas áreas do conhecimento e, consequentemente, pode adotar ferramentas interdisciplinares para seus procedimentos analíticos. Para além das investigações usuais já supracitadas (e.g. valores epistêmicos, visualização, racionalidade, argumentação, experimentação etc.), nesta seção foco somente em alguns exemplos investigativos onde a filosofia da ciência pode se aproximar não apenas da prática, como pode também ser socialmente informada sem perder seu teor filosófico.

Em estudos de casos onde os pesquisadores lidam com conservação, problemas ambientais, mudanças climáticas, epidemias, entre outros é comum que os cientistas tenham de interagir com comunidades locais e tradicionais e tomadores de decisão - configurando assim práticas inter- e transdisciplinares. Alguns exemplos de contribuições filosóficas neste contexto advêm tanto em dar assistência a tomadores de decisão para lidar com temas de investigação controversos como, por exemplo, poluição ambiental (KOSOLOSKY, 2012, ver entrevista com Kevin Elliot). O The Toolboxproject $^{6}$, coordenado pelo prof. Michael O'Rourke, na Universidade Estadual de Michigan, é um exemplo extremamente interessante de uma iniciativa onde a filosofia, em colaboração interdisciplinar ativa e conjunta com a ciência, realiza oficinas informativas. Bastante comum são os estudos que se debruçam sobre a dinâmica de colaboração entre os cientistas dentro dessas práticas inter- e transdisciplinares, seguem alguns exemplos. Andersen (2016) descreve diferentes tipos de atividades de pesquisa determinados por certos tipos de valores na ciência, e discorre sobre os mecanismos subjacen-

${ }^{6}$ O Toolbox project pode ser acessado pela plataforma: http://tdi.msu.edu/. 
tes às tensões destas práticas interdisciplinares e colaborativas. Wagenknecht (2015) investiga as relações que surgem dentro dessas práticas como dependência epistêmica e vigilância epistêmica entre os participantes. Freeth e Caniglia (2020) chamam a atenção aos desafios dos processos colaborativos e desenvolvem uma estratégia para pesquisadores aprenderem a colaborar ao mesmo tempo em que colaboram, assim criam espaços para aprendizagens possíveis e desenvolvem capacidades colaborativas como orientações, conhecimentos e habilidades.

Por fim, existe uma reintegração entre diversos campos e abordagens em que o objetivo é encorajar investigações de uma vasta gama de campos científicos utilizando toda a capacidade metodológica e teórica disponível. A ideia é incentivar reflexões sobre as ciências, e suas práticas, incluindo limitações e prospectos de aprendizagem com outras tradições disciplinares devotadas ao estudo da ciência, como a sociologia da ciência, estudos em ciência e tecnologia, entendimento público da ciência e história da ciência (ANKENY et al., 2011).

\section{Considerações finais}

Há quem afirme que a filosofia "em seu encastelamento universitário [...] ficou demasiadamente nobre; em sua linguagem altamente especializada, ficou distante da vida diária e ordinária; na soberba de seus mestres, ficou estéril” (QUINHONES \& COSTA, 2010, p. 148, ecoando Marc Sautet em sua obra Um café para Sócrates, 2003). Concordando ou não com esta afirmação pungente, é possível reconhecer que parte das investigações filosóficas ainda permanecem sendo inferências abstratas (MURCHO, 2006; QUINHONES \& COSTA, 2010). Com exceção de trabalhos recentes sobre modelos e experimentação (bem como outros domínios não abstratos e aplicáveis) que necessitam de um profundo detalhamento do processo científico, a prática científica propriamente dita continua quase completamente ignorada pela filosofia da ciência e filosofia analítica anglófona. A Sociedade de Filosofia da Ciência em Prática surge, então, com a intenção de mitigar esta situação promovendo estudos meticulosos e sistemáticos da prática científica sem deixar de considerar verdade e racionalidade (ANKENY et 
al., 2011). Assim, a filosofia da ciência em prática objetiva uma epistemologia das práticas científicas focando nas possíveis construções e utilizações do conhecimento epistêmico. Visa uma compreensão da ciência que evita tanto a crença de que a objetividade do conhecimento pode ser garantida por uma justificação do conhecimento que elimina o papel dos cientistas, quanto uma mera interpretação psicológica e sociológica atrelada à subjetividade dos cientistas (BOON, 2017).

A ideia deste artigo foi apresentar ao leitor uma terceira perspectiva de pesquisa para a filosofia da ciência. Naturalmente, algumas questões irão surgir como por exemplo, o quanto de filosofia deve olhar para a prática; e, se esse olhar empírico não acarretaria riscos à generalidade em detrimento de uma fragmentação ou especialização (BOUMANS \& LEONELLI, 2013). Não é defendido aqui que todas investigações filosóficas sobre a ciência precisam necessariamente se debruçar sobre a prática científica ou que a filosofia da ciência em prática precisa ser empírica. É importante ter em mente que a maior parte dos estudos de casos concretos sobre a prática científica são baseadas em métodos, princípios e interpretações advindos de estudos de casos teóricos ou históricos. Assim, tópicos gerais em filosofia da ciência (e.g. experimentação, teoria, evidência, realismo, reducionismo, valores, etc.) são tanto debatidos em detalhes como subsídios teóricos necessários para uma filosofia da ciência em prática (RADDER, 2012).

Me fazendo dos clichês ao começar este artigo com um fragmento do poema Auguries of Innocence de William Blake, penso na alegoria para a filosofia da ciência em uma alusão à possibilidade de um olhar minucioso aos pormenores da prática científica que por muito foi ignorado e tido como secundário. Podemos perceber uma infinitude de discussões e investigações atreladas e associadas às práticas científicas às quais a filosofia pode estar ativamente inserida, beneficiar e ser beneficiada. Importante notar que as palavras abaixo podem não ecoar a voz de demais colegas praticantes, no entanto, tomo a liberdade de relembrar Cupani (data incerta), quando nos chama atenção para a ambiguidade da expressão "filosofia da ciência" - podendo referir-se tanto à reflexão epistemológica sobre o conhecimento científico quanto à reflexão mais ampla sobre o sentido existencial do papel político desta atividade. Se tomarmos nosso atual contexto brasileiro, onde 
as humanidades são áreas do saber ameaçadas de extinção em esdrúxulas tentativas de as tornarem terra incognita, pautar a filosofia da ciência em prática, nas suas mais diversas formas, não é somente uma questão de quais abordagens de pesquisa escolher, mas sim um posicionamento político contra um governo escancaradamente fascista. Encerro, muito longe de ter exaurido a cornucópia que é a filosofia da ciência em prática, mas espero ter contribuído para o começo de uma epifania em que a filosofia da ciência não mais se isole em investigações metateóricas, mas que se redefina, tornando-se empiricamente engajada e socialmente informada.

\section{Agradecimentos:}

Agradeço ao CNPQ (código de financiamento 465767/2014-1) e à CAPES (código de financiamento 23038.000776/2017-54) pelo apoio concedido ao INCT-INTREE. Este artigo foi beneficiado por comentários de Bruno Althoff e Pedro Bravo, aos quais sou grata.

\section{Referências}

ANDERSEN, Hanne. The second essential tension: on tradition and innovation in interdisciplinary research. Topoi 32, pp. 3-8, 2013.

ANDERSEN, Hanne. Collaboration, interdisciplinarity and the epistemology of contemporary sciences. Studies of History and Philosophy of Science Part A 56, pp. 1-10, 2016.

ANKENY, Rachel; CHANG, Hasok; BOUMANS, Marcel \& BOON, Mieke. Introduction: philosophy of science in practice. European Journal for Philosophy of Science 1(3), pp. 303-307, 2011.

AUFRECHT, Monica. The context distinction: controversies over feminist philosophy of science. European Journal for Philosophy of Science, 1, n. 373, 2011.

AYMORÉ, Débora; KOIDE, Kelly \& FERREIRA, Mariana Toledo. Ativismo, feminismo e filosofia da ciência: entrevista com Helen Longino. Scientiae Studia, São Paulo, vol. 15, n. 1, pp. 145-162 2017.

BARBEROUSSE, Anouk; BONNAY, Dennis \& COZIC, Mikael. Précis de philosophie des sciences. Paris: Vuibert, 2011. 
BOON, Mieke. Philosophy of science in practice: a proposal for epistemological constructivism. In LEITGEB, $\mathrm{H}$; NINILUOTO, I; SEPPÄLÄ, P. \& SOBER, E. (eds.) Logic, methodology and philosophy of science, pp. 289-310. Proceedings of the 15th International Congress (CLMPS 2015): College Publications, 2017.

BOUMANS, Marcel. \& LEONELLI, Sabina. Introduction: on the philosophy of science in practice. Journal of General Philosophy of Science 44, pp. 259-261, 2013.

BOURDIEU, Pierre. Os usos sociais da ciência. São Paulo: Editora UNESP, [1997]2004.

BRAGA, Marco; GUERRA, Andreia \& REIS, José Claudio. Breve história da ciência moderna. Vol. 1: Convergência de saberes (Idade Média). Rio de Janeiro: Zahar, 2003.

BRAGA, Marco; GUERRA, Andreia \& REIS, José Claudio. Breve história da ciência moderna. Vol. 2: Das máquinas do mundo ao Universo-máquina (séc. XV a XVII). Rio de Janeiro: Zahar, 2004.

BRAGA, Marco; GUERRA, Andreia \& REIS, José Claudio. Breve história da ciência moderna. Vol. 3: Das luzes ao sonho do doutor frankenstein (séc. XVIII). Rio de Janeiro: Zahar, 2005.

BRAGA, Marco; GUERRA, Andreia \& REIS, José Claudio. Breve história da ciência moderna. Vol. 4: A belle-époque da ciência (séc. XIX). Rio de Janeiro: Zahar, 2007.

BRYSON, Anthony Alan. The view from the armchair: a defense of traditional philosophy. $\mathrm{PhD}$ (Doctor of Philosophy) thesis, University of Iowa, 2009.

CAPONI, Gustavo. Leyes sin causa y causas sin ley en la explicación biológica. Bogotá: Universidad Nacional de Colombia, 2014.

CHANG, Hasok. Epistemic activities and systems of practice: units of analysis in philosophy of science after the practice turn. In SOLER, L; ZWART, S; LYNCH, M. \& ISRAEL-JOST, V. (eds.) Science after the practice turn in the philosophy, history and social studies of science. New York: Routledge Studies in the Philosophy of Science, 2014.

CID, Rodrigo. Leis da Natureza: uma abordagem filosófica. Macapá: UNIFAP.

CRAVER, Carl. Explaining the brain: mechanisms and the mosaic unity of neurosciences. New York: Oxford University Press, 2007.

CUPANI, Alberto. Formación científica y reflexión filosófica (Acerca de la utilidad de la filosofía de la ciencia en la formación del científico). Práctica 
Científica y Reflexión Filosófica. (Acessado em:

https://www.unrc.edu.ar/publicar/cde/05/Cupani.htm).

DASTON, Lorraine. \& GALISON, Peter. Objectivity. New York: Zone Books, 2010.

DE OLIVEIRA, Marco Barbosa. On the commodification of science: the programmatic dimension. Science \& Education 22, pp. 2463-2483, 2013.

DE REGT, Henk. Understanding scientific understanding. New York: Oxford University Press, 2017.

DE REGT, Henk \& DIEKS, Dennis. A contextual approach to scientific understanding. Synthese 144, pp.137-170, 2005.

DUPRÉ, John. The disorder of things. Cambridge: Harvard University Press, 1993.

EL-HANI, Charbel Niño \& LUDWIG, David. Philosophy of ethnobiology: understanding knowledge integration and its limitation. Journal of Ethnobiology, vol. 39, 2019.

FEYERABEND, Paul. Adeus à razão. São Paulo: Editora UNESP, [1987]2010.

FODOR, Jerry. Special sciences (or: the disunity of science as a working hypothesis). Synthese, pp. 97-115, 1974.

FRANK, Phillip. Philosophy of science: the link between science and philosophy. Upper Saddle River, NJ, US: Prentice Hall/Pearson Education, 1957.

FREETH, Rebecca. \& CANIGLIA, Guido. Learning to collaborate while collaborating: advancing interdisciplinary sustainability research. Sustainability Sciences 11, n.3, 2020.

GALISON, Peter. How experiments end. Chicago: The University of Chicago Press, 1987.

GALISON, Peter \& STUMP, David (eds.) The disunity of science: boundaries, contexts and power. Palo Alto, California: Stanford University Press, 1996.

HACKING, Ian. Representar e intervir: tópicos introdutórios de filosofia da ciência natural. Rio de Janeiro: EDUERJ, [1983]2012.

HEMPEL, Carl \& OPPENHEIM, Paul. Studies in the logic of explanation. Philosophy of Science 15, pp. 135-175, 1948. 
KHALIFA, Kareem. Understanding, explanation and scientific knowledge. Cambridge: Cambridge University Press, 2017.

KITCHER, Philip. Explanatory unification. Philosophy of Science 48, pp. 507-531, 1981.

KNUUTTILA, Tarja. Modelling and representing: an artefactual approach to model-based representation. Studies in History and Philosophy of Science, 42, pp. 262-271, 2011.

KOIDE, Kelly; FERREIRA, Mariana Toledo \& MARINI, Marisol. Arqueologia e a crítica feminista da ciência: entrevista com Alison Wylie. Scientiae Studia, São Paulo, vol. 12, n. 3, pp. 5449-590, 2014.

KOSOLOSKY, Lazlo. Philosophy-of-science in practice vs. philosophy of science-in-practice. Newsletter SPSP, Winter , pp. 9-10, 2012.

KUHN, Thomas. A estrutura das revoluções científicas. 5a. ed. São Paulo: Editora Perspectiva S.A, [1962]1997.

KUSCH, Martin. A socially-engaged philosophy, 2020. Disponível em: $<$ https://www.youtube.com/channel/UCyGsq118XnjrHhpgLCea6kg/videos $>$

LACEY, Hugh. Valores e a atividade científica. São Paulo: Editora Discurso, 1998.

LACEY, Hugh. Valores e a atividade científica 1. São Paulo: Associação Filosófica Scientia Studia/Editora 34, 2008.

LACEY, Hugh. Valores e a atividade científica 2. São Paulo: Associação Filosófica Scientia Studia/Editora 34, 2010.

LADYMAN, James. Understanding philosophy of science. Abingdon: Routledge, 2001.

LADYMAN, James. The history of philosophy of science. In. BECKER, K. \& THOMPSON, I.D. (eds.) The Cambridge History of Philosophy, 1945 2015, pp. 189-209. Cambridge University Press, 2019.

LAKATOS, Inre. La metodología de los programas de investigación científica. Madrid: Alianza, 1987.

LEONELLI, Sabina. Data-centric biology: a philosophical study. Chicago: The University of Chicago Press, 2016.

LEONELLI, Sabina \& TEMPINI, Niccolò. (eds.) Data journeys in the sciences. London: Springer, 2020. 
LONGINO, Helen. Science as social knowledge: values and objectivity in scientific inquiry. Nova Jersey: Princeton University Press, 1990.

LUDWIG, David \& POLISELI, Luana. Relating traditional and academic ecological knowledge: mechanistic and holistic epistemologies across cultures. Biology \& Philosophy, vol. 33, n. 43, 2018.

MAYR, Ernst. O desenvolvimento do pensamento biológico: diversidade, evolução e herança. Brasília: UNB, [1982]1998.

MORGAN, Mari \& MORRISON, Margareth (eds). Models as mediators: perspectives on natural and social sciences. Cambridge: Cambridge University Press, 1999.

MURCHO, Desidério. Does science need philosophy? Rev. Eletr. Infor. e Cogn., v. 5, n.2, pp. 50-58, 2006.

OLIVEIRA, Marco Barbosa. Sobre o significado político do positivismo lógico. Crítica Marxista, São Paulo: Boitempo, v. 1, n. 14, pp. 73-84, 2002.

OLIVEIRA, Tiago. Notas sobre o problema do realismo científico. Investigação Filosófica, v. 10, n. 2, pp. 47-59, 2019.

PICKERING, Andrew. (ed.) Science as practice and culture. Chicago and London: The University of Chicago Press, 1992.

POLISELI, Luana. When ecology and philosophy meet: constructing explanation and assessing understanding in scientific practice. Tese (Doutorado em Ensino, Filosofia e História das Ciências) - Universidade Federal da Bahia, Salvador, 2018.

POLISELI, Luana \& LEITE, Clarissa Machado Pinto. Modelling transdisciplinary practices: an interplay between disagreement and trust. In. LUDWIG, D; KOSKINEN, I; POLISELI, L; MNCUBE, Z. \& REYESGALINDO, L. (eds.) Global epistemologies and philosophies of science. Routledge, Taylor \& Francis Group, 2020 (no prelo).

POPPER, Karl. A lógica da pesquisa científica. São Paulo: Cultrix, [1959]1993.

PSILLOS, Stathis. Scientific realism: how science tracks the true? New York: Routledge, 1999.

PSILLOS, Stathis. What is general philosophy of science? Journal of General Philosophy of Science, n. 43, pp. 93-103, 2012.

QUINHONES, Dionatans Godoy \& COSTA, Márcio Luis. Filosofia: uma ciência prática? Multitemas, n. 38, pp. 145-159, jul., 2010. 
RADDER, Hans. The commodification of academic research: science and the modern university. Pittsburgh: University of Pittsburgh Press, 2010.

RADDER, Hans. What prospects for a general philosophy of science? Journal for General Philosophy of Science 43(1), pp. 89-92, 2012.

SALMON, Wesley. Scientific explanation and the causal structure of the world. Princeton: Princeton University Press, 1984.

SHAPERE, Dudley. Reason and the search for knowledge: investigations in the philosophy of science. Boston Studies in the Philosophy of Science, v. 78. Drodrecht: D. Reidel Publishing Company, 1984.

SHINN, Terry \& RAGOUET, Pascoal. Controvérsias sobre a ciência: por uma sociologia transversalista da atividade científica. Coleção Estudos sobre a Ciência e Tecnologia. São Paulo: Associação Nacional Scientia Studia/Editora 34, 2008.

SOCIETY FOR PHILOSOPHY OF SCIENCE IN PRACTICE. Mission statement. Disponível em:

$<$ https://www.philosophy-science-practice.org/about/mission-statement $>$.

Acessado em: 13/06/2020.

SOLER, Léna. Tacit aspects of experimental practices: analytical tools and epistemological consequences. European Journal for Philosophy of Science 1, n. 393, 2011.

SOLER, Léna; ZWART, Sjoerd; LYNCH, Michael \& ISRAEL-JOST, Vincent (eds.) Science after the practice turn in the philosophy, history and social studies of science. New York: praticantes da psp, I am aware that might be the case of my opinion in here might differ from those psp practioners, even tought, I believeRoutledge Studies in the Philosophy of Science, 2014.

SRPOISE, The Consortium for Socially Relevant Philosophy of/in Science and Engineering. Disponível em: <http://srpoise.org/>. Acessado em 10/07/2020.

STERN, David. The practical turn. In TURNER, S.P. \& ROTH, P.A. (eds.) The Blackwell Guide to the philosophy of social sciences, pp. 185-206. Oxford: Blackwell Publishing Ltd, 2003.

THE TOOLBOX PROJECT. Dialogue initiative. Disponível em: $<$ http://tdi.msu.edu/>. Acessado em: 15/07/2020.

WAGENKNECHT, Susan. Facing the incompleteness of epistemic trust: managing dependence in research practice. Social Epistemology 29(2), pp. 160-184, 2015. 